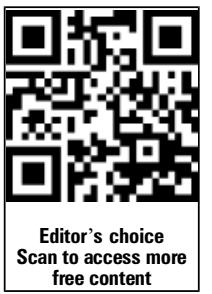

free content

\title{
Neutropenic sepsis: prevention and management of neutropenic sepsis in cancer patients (NICE Clinical Guideline CG151)
}

\author{
Jessica Bate, ${ }^{1}$ Faith Gibson, ${ }^{2,3}$ Emma Johnson, ${ }^{4}$ Karen Selwood, ${ }^{5}$ \\ Roderick Skinner, ${ }^{6}$ Julia Chisholm ${ }^{7}$
}

${ }^{1}$ Division of Clinical Sciences, Paediatric Infectious Diseases Research Group, St. George's, University of London, London, UK

${ }^{2}$ Department of Paediatric Oncology, Great Ormond Street Hospital NHS Foundation Trust, London, UK

${ }^{3}$ London South Bank University, London, UK

${ }^{4}$ Oncology Unit, Royal Hospital for Sick Children, Edinburgh, UK ${ }^{5}$ Oncology Unit, Alder Hey Childrens NHS Foundation Trust, Liverpool, UK

${ }^{6}$ Department of Paediatric and Adolescent Oncology, Newcastle upon Tyne Hospitals NHS Foundation Trust, Newcastle upon Tyne, UK

${ }^{7}$ Children and Young People's Unit, Royal Marsden NHS Foundation Trust, Sutton, UK

\section{Correspondence to} Dr Jessica Bate, Division of Clinical Sciences, Paediatric Infectious Diseases Research Group, St. George's, University of London, London SW17 ORE, UK; jbate@sgul.ac.uk

Received 8 January 2013 Revised 6 February 2013 Accepted 8 February 2013
To cite: Bate J, Gibson F, Johnson $\mathrm{E}$, et al. Arch Dis Child Educ Pract Ed 2013;98:73-75.
Information about current guideline In September 2012, the National Institute for Clinical Excellence (NICE) published a guideline entitled 'Neutropenic sepsis: prevention and management of neutropenic sepsis in cancer patients. ${ }^{1}$ The aim of the guideline is to 'improve outcomes by providing evidence-based recommendations on the prevention, identification and management of this life-threatening complication of cancer treatment' for children, young people and adults. The National Collaborating Centre for Cancer was commissioned by NICE to develop the guideline and establish a Guideline Development Group, including children and young people's cancer nurses and consultants, which reviewed the evidence and developed the recommendations.

\section{Previous guideline}

There are no previously published national guidelines for the management of neutropenic sepsis in children. In July 2008, the Children's Cancer and Leukaemia Group (CCLG) produced a framework document for the treatment of febrile neutropenia, focusing on the definitions of fever and neutropenia, and on developing a practical management strategy for low-risk patients, which individual centres could incorporate into local policies. The document was produced in response to a survey suggesting wide variation in the definitions and management of febrile neutropenia, following an exhaustive literature review, small group discussion and a national Delphi consensus process. ${ }^{2}{ }^{3}$ A recent audit of UK paediatric oncology centres revealed that current practice differs widely from recommendations contained within the CCLG framework. ${ }^{4}$

Controversial and key issues that the guideline addresses
- Definition of febrile neutropenia: Neutrophils $0.5 \times 10^{9} / 1$ or lower and temperature higher than $38^{\circ} \mathrm{C}$, including one isolated fever. Children with fever should always be treated with intravenous antibiotics if neutrophils are $0.5 \times 10^{9} / 1$ or lower. If unwell, the child should be treated with intravenous antibiotics regardless of neutrophil count or temperature.

- Appropriate investigations: In addition to routine full blood count, kidney and liver function tests (including albumin), C-reactive protein (CRP) and venous lactate levels should also be measured. A peripheral blood culture (when there is a central venous access in situ) should be obtained in addition to a central venous blood culture. Children less than 5 years old should have urinalysis sent. Chest radiograph should only be performed if clinically indicated.

- Risk assessment of septic complications: A validated scoring system should be used to assess a child's risk of septic complications. This is the modified Alexander rule in paediatric practice (see box 1).

- Antibiotic treatment: $\beta$ lactam monotherapy (eg, piperacillin-tazobactam) rather than dual therapy with an aminoglycoside (eg, gentamicin). Aminoglycosides should not be given unless there are patientspecific or local microbiological indications. However, factors such as local antibacterial resistance patterns and individual patient drug allergy may determine that the use of piperacillin-tazobactam monotherapy is not appropriate.

- There is too little evidence to recommend the use of either antibiotic prophylaxis or routine G-CSF (granulocyte-colony stimulating factor) in children to prevent neutropenic sepsis.

- Empiric glycopeptide antibiotics (eg, vancomycin, teicoplanin) should not be offered to patients with suspected 
Box 1 Modified Alexander rule for children and young people $<18$ years old as risk assessment for septic complications. ${ }^{5}$

- Cancer patients are at low risk of septic complications unless one or more of the following conditions apply:

- Treatment for acute myeloblastic leukaemia or Burkitt lymphoma

- Induction phase of treatment for acute lymphoblastic leukaemia

- Progressive disease; or treatment for relapsed disease with marrow involvement

- Presenting with any of the following features:

- hypotension

- tachypnoea

- hypoxia-defined as saturations less than $94 \%$ in air

new changes in chest radiography

altered mental status

severe mucositis

vomiting or abdominal pain

focal infection

- other clinical reason(s) for inpatient treatment neutrophil count $<0.1 \times 10^{9} /$ I

neutropenic sepsis who have central venous access devices unless there are patient-specific or local microbiological indications.

- Initial empiric antibiotics in patients with unresponsive fever should not be changed unless there is clinical deterioration or a microbiological indication. This does not apply to the addition of empirical antifungal treatment.

- A switch from intravenous to oral antibiotic therapy should be considered after $48 \mathrm{~h}$ of treatment in patients whose risk of developing septic complications has been reassessed as low using a validated risk scoring system (box 1). No recommendations are made on duration of oral antibiotic therapy after switching from intravenous therapy.

- Consider outpatient therapy in patients reassessed as low risk.

\section{Underlying evidence base/methodology}

NICE recommendations are based on systematic reviews of the best available evidence. When evidence is limited, the Guideline Development Group make recommendations based on their experience and opinion of what constitutes good practice (see box 2 and full evidence review at (http://www.nice.org.uk/ nicemedia/live/12349/58165/58165.pdf)).

\section{What do I need to know?}

- What should I stop doing?

- Stop giving aminoglycosides routinely as 'double Gram-negative cover'

- Stop adding teicoplanin/vancomycin routinely to 'cover the line'

\section{Box 2 Resources}

http://guidance.nice.org.uk/CG151

Link to NICE guideline and full guideline

- http://guidance.nice.org.uk/CG151/Publiclnfo/pdf/

English Link to public information on neutropenic sepsis guidance

h http://www.nice.org.uk/newsroom/podcasts/index.jsp? pid $=45$

Neutropenic sepsis: prophylaxis podcast with Dr Bob Phillips

- Stop doing chest radiographs routinely-only if clinically indicated

- What should I ensure I've started doing?

- Taking a peripheral blood culture at presentation

- Taking blood for venous lactate level at presentation

- Routinely checking CRP and albumin at presentation

- Sending urine of children less than 5 years of age

- Considering switching to oral antibiotics at $48 \mathrm{~h}$ if assessed as low risk

- Considering outpatient therapy after $48 \mathrm{~h}$ if assessed as low risk

- Ensure families are well informed about febrile neutropenia (box 3)

- What can I continue to do as before?

- Treat any child who is clinically unwell with suspected sepsis with antibiotics, regardless of neutrophil count or temperature

- Ensure children are assessed by a healthcare professional with competence of managing complications of anticancer treatment

- Manage all patients as inpatients for the first $48 \mathrm{~h}$

- Ensure ongoing surveillance of antimicrobial resistance patterns in your centre

What should I do differently?

- The threshold for starting treatment with intravenous antibiotics is a neutrophil count of $0.5 \times 10^{9}$ cells/ 1 or lower together with a temperature of higher than $38^{\circ} \mathrm{C}$.

\section{Unresolved controversies}

There is sparse evidence for very early (before $24 \mathrm{~h}$ ) discharge for low-risk patients with neutropenic sepsis. Research is recommended to investigate whether a shorter hospital admission is safe and effective for selected patients.

Teenagers and young adults (TYA) are more likely to have neutropenic septic deaths than other age groups, so it is essential that there is a heightened vigilance of this patient group. Although not included as a defined subgroup in the research recommendations from this NICE guidance, there should be a specific focus on improving outcomes in the TYA group by conducting high-quality research.

Clinical bottom line 
Box 3 What do I need to do to inform the families in my care?

Explain neutropenic sepsis and the possible approaches to treatment.

- Ensure age appropriate information is provided for children and young people

- Where appropriate, work with the play specialist to ensure young children have understood what it means

- With young people, often nursing and medical textbooks and associated diagrams can help explain neutropenia

- Similar approaches can be used with families who are not fluent in English

- Ensure information is continually built upon and reinforced at each discharge.

- Provide sufficient information so that families can be involved in decisions about care.

- Ensure families know what to do when at home, ensure they have a thermometer, and have a clear plan of what to do when they are concerned, and know whom they should contact in an emergency.

- Provide details of the action plan in the family held record/patient treatment summary.

- Families should have all the information they need to inform new healthcare teams of the care they are anticipating based on signs and symptoms.
Febrile neutropenia is a well-recognised cause of death in children with cancer. Great care must be taken to manage such patients appropriately.

This NICE guidance contains evidence-based recommendations for the management of febrile neutropenia, and for the safe reduction of therapy in selected patients following risk stratification.

Contributors JB: Conception and design. JB, FG, EJ, RS and JC: Manuscript draft, revision and final approval.

Competing interests None.

Provenance and peer review Commissioned; externally peer reviewed.

\section{REFERENCES}

1 National Institute for Health and Clinical Excellence. Neutropenic sepsis: prevention and management of neutropenic sepsis in cancer patients. CG151 London: National Institute for Health and Clinical Excellence, 2012.

2 Phillips R, Selwood K, Lane SM, et al. Variation in policies for the management of febrile neutropenia in United Kingdom Children's Cancer Study Group centres. Arch Dis Child 2007;92:495-8.

3 Gibson F, Chisholm J, Blandford E, et al. Developing a national 'low risk' febrile neutropenia framework for use in children and young people's cancer care. Support Care Cancer 2012 Dec 20 (Epub ahead of print)

4 Bate J, Gibson F, Selwood K, et al. A reaudit of current febrile neutropenia practice in UK paediatric oncology centres prior to implementation of NICE guidance. Arch Dis Child 2013;98:315-16.

5 Alexander SW, Wade KC, Hibberd PL, et al. Evaluation of risk prediction criteria for episodes of febrile neutropenia in children with cancer. J Pediatr Hematol Oncol 2002;24:28-42. 